\title{
A numerical modeling of natural gas using multistage membrane permeation
}

\author{
Muhammad Ahsan a , Arshad Hussain \\ ${ }^{a}$ School of Chemical \& Materials Engineering, National University of Sciences \& Technology, H-12, \\ Islamabad, 44000, Pakistan \\ Email:ahsan@scme.nust.edu.pk
}

\begin{abstract}
In this paper, a mathematical model is presented that has been developed using membrane permeation for the recovery of helium from natural gas in hollow fiber modules. Recovery of helium from natural gas is an important process, so investigating a robust and accurate numerical model is of great significance for membrane gas separation industry. The numerical approach described in this paper illustrates the correct separation of helium by a membrane module with minimal computational effort. The model has also been applied for multi-stage membrane permeation to check its flexibility at various operating conditions. To our knowledge, this is the first reliable numerical investigation for the recovery of helium from a gas mixture, consists of six components by using multistage membrane permeation with countercurrent flow pattern. The mathematical modeling comprising six components is a complex and demanding research task in terms of the computational programming required for its solution. Broyden's method and Paterson approximation are used in this paper to deal with the computational complexities of the numerical model. Membrane stem structures and operating conditions are easy to improve with this numerical model because of its algebraic nature and simplicity.
\end{abstract}

Keywords: $\quad$ Numerical modeling, membrane gas separation, countercurrent, multistage permeation 


\section{INTRODUCTION}

Membrane gas separation has been used progressively in the process industry during the last three decades. Development of the asymmetric membranes is the reason for this, which combines high selectivity and high permeability. Fritzsche and Narayan (Fritzsche \& Narayan, 1987) present an analysis of the application of different systems in hydrogen, carbon dioxide-methane, and oxygen-nitrogen separations. Membrane separation processes normally give products of intermediate purity up to $96 \%$. Besides these numerous studies have shown that for low to medium level production membrane separation can be a good alternative (Meindersma, 1990; Scholz, Alders, Lohaus, \& Wessling, 2015; Spillman, 1989). Membrane systems are preferred in offshore installations due to their fewer area requirements and low weight. Economical processes can be designed by combining membrane process with cryogenic distillation or physical adsorption (Lock, Lau, \& Shariff, 2015; Stookey, Patton, \& Malcolm, 1986). A spiral wound membrane modules design model was given by Hogsett et. al. (Hogsett \& Mazur, 1983) for the permeation calculations occur due to multicomponent gases. A group of algebraic equations can be driven by the performance of membrane (Weller \& Steiner, 1950) by supposing complete mixing of the gas on the permeate and feed side of the membrane. An explanation for binary mixtures and counter-current permeators has been presented by Boucif et. al. (Boucif, Majumdar, \& Sirkar, 1984). This model tends to overrate the performance of a module and is also stated to be less accurate for certain cases of stage cut and area (Rautenbach \& Dahm, 1986). Approximate analytic solutions have been derived by several researchers for binary mixtures and countercurrent permeators. Such models have been assessed to be very complex in terms of initial estimates and too inaccurate from a convergence perspective (Barnicki \& Fair, 1992). An estimated solution, effective for binary mixtures has been derived in a research paper by Krowidi et al. (Krovvidi, Kovvali, Vemury, \& Khan, 1992). This numerical approach has been equated with a series solution (Boucif et al., 1984) and shown to provide improved data compared with the series solution. To overcome the numerical difficulties in solving the boundary value problems, numerous changes, and different numerical methods have been suggested to solve the model equations (Chowdhury, Feng, Douglas, \& Croiset, 2005; Coker, Freeman, \& Fleming, 1998; Kaldis, Kapantaidakis, \& Sakellaropoulos, 2000). These models involve the introducing additional assumptions and simplifying the governing equations that lead to estimated solutions involving less computational time and effort. In the case of binary gas mixtures, the governing equations are solved by asymptotic solutions in a cross-flow approach depended for the Navier-Stokes equations. But, these methods do not actually ease the numerical challenges. At low permeabilities, the results look to be inaccurate (Ahmad et al., 2015; Basaran \& Auvil, 1988). The present work is a novel contribution to the mathematical modeling for the recovery of helium from a gas mixture (Ahsan \& Hussain, 2015). There are two major novelties in the presented model: (1) Broyden's method has been used in this research instead of a conventional Newton-Raphson method. In addition, from a speed advantage, Broyden's method is simpler to configure. Because Newton-Raphson uses the perturbations on every iteration, it is sensitive to the values chosen for the perturbation size. If the perturbation is too small or too big, the method may take much longer to converge on a solution or may not converge at all (Kvaalen, 1991). (2) Paterson approximation has been used to calculate mean partial pressure difference across the membrane instead of the traditional log mean formula (Paterson, 1984). Mathematical modeling of helium recovery from natural gas is a complex problem due to a small mole fraction of helium present in natural gas. The conventional numerical techniques were unable to model this problem successfully. The last study in this area was done in 1983 by C. Pan (C. Y. Pan, 1983). In this research, we have applied our mathematical model (based on Broyden's method and Paterson approximation) (Kvaalen, 1991; Paterson, 1984) to recover helium from a gas mixture. The study attempts to model the recovery of helium gas from six component natural gas using multistage membrane permeation. The natural gas considered in this study is composed of $\mathrm{He}, \mathrm{CO}_{2}, \mathrm{~N}_{2}, \mathrm{CH}_{4}, \mathrm{C}_{2} \mathrm{H}_{6}$, and $\mathrm{C}_{3} \mathrm{H}_{8}$. To the best of our knowledge based on the published literature, our work is the first attempt in this area of research. The numerical model is implemented for a countercurrent flow configuration with various operating conditions and has been found to be very accurate for helium recovery from multicomponent natural gas. The numerical model is used to study dynamic performance of different membrane module configurations for helium enrichment and recovery from natural gas. Several operational modes for countercurrent flow pattern are also studied to increase the helium enrichment and recovery in the permeate stream.

\section{NUMERICAL MODELING}

The objective of the research described here has been to formulate and implement a fast and simple numerical model for gas separation by membrane separation. This part shows the formulation of the numerical model and the assumptions used in it. A single fiber schematic hollow fiber membrane module is shown in Figure 1 for the countercurrent flow pattern. In solution-diffusion model Fick's law is used to express the local gas permeation (Pettersen \& Lien, 1994): 
Ahsan et al., A numerical modeling of natural gas using multistage membrane permeation

$$
d n_{i}=\frac{F_{i} d A\left(p_{f i}-p_{p d}\right)}{d}
$$

The area of a membrane needed for a specific separation value can be calculated up to a higher level of accuracy. Non-ideal behavior in the gas phase was explained by using Fugacity coefficients (Fattah, Hamam, Alenezi, Ettoueny, \& Hughes, 1992). The composition and pressure dependence was shown by implementing dual mode transport

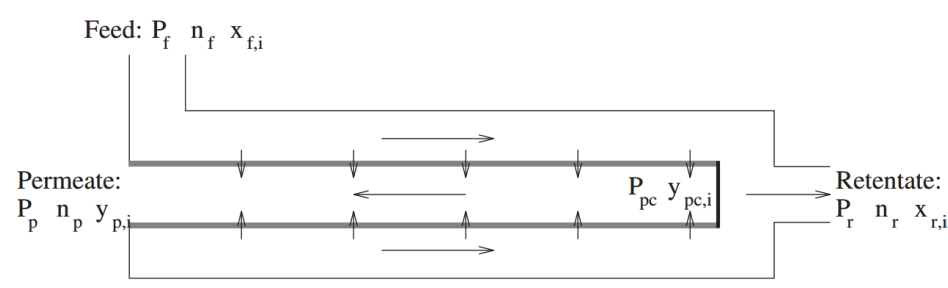

Figure 1. Schematic of hollow fiber membrane module model in the permeabilities of the gas mixture (Chern, Koros, \& Fedkiw, 1985). The pressure loss inside the hollow fibers module was described by implementing Hagen-Poiseuille equation in the differential form (C.Y. Pan \& Habgood, 1978). In a hollow fiber membrane module, the following model dependent on equations presented in a differential form for the separation of a binary mixture (Rautenbach \& Dahm, 1986):

$$
\begin{aligned}
& \frac{d F}{d T}=-R[(x-\delta y)+s[(1-x)-\delta(1-y)]] \\
& \bar{F} \frac{d x}{d I}=-R[(1-x)(x-\delta y)-s x[(1-x)-\delta(1-y)]]
\end{aligned}
$$

As shown in Figure 2, the component and mass balances of feed side of the membrane module are described by equations (2) and (3). The dimensionless membrane area, $\mathrm{R}$ is expressed as

$$
R=\frac{A F q_{2}}{n_{f}}
$$

This model is dependent on the suppositions of trivial axial diffusion in permeate and feed side, minor pressure losses at the feed side and plug flow at permeate and feed side. Membrane permeation is never reached at a rate governed processequilibrium. Rate governed methods

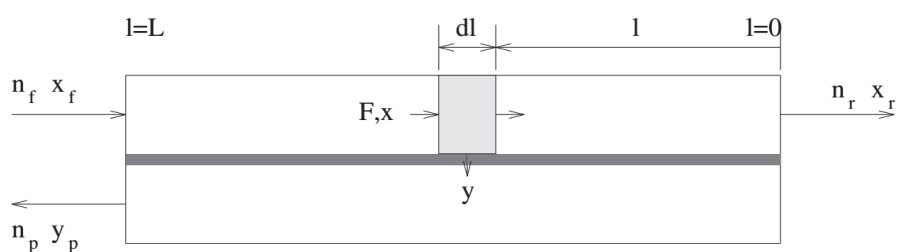

Figure 2. Cross section area of a hollow fiber module perhaps normally are defined by associations between a flux and a driving force. The relation in driving force and flux is defined by the renowned integral equation,

$$
Q=U A \Delta T_{\mathrm{m}}
$$

which uses logarithmic mean temperature difference to describes the heat exchanger duty, the heat transfer area, and the heat transfer coefficient. Integral relation among differences in partial pressure and flux across the membrane can be shown as

$$
\int d n_{i}=Q_{i} A \Delta P_{n_{i}, i}
$$

Equation (7) is used to express $P_{m, i}$, considering module operating under counter-current flow

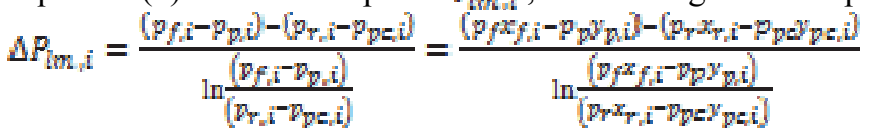

Correct data about the active thickness of commercial membranes modules used for gas separation is normally not accessible. For membrane module, the component material balances are shown by equation (8).

$$
\begin{aligned}
& n_{f} x_{f i}=n_{p} y_{p i}+n_{r} x_{r i} \\
& \int d r_{i}=n_{r} y_{i}
\end{aligned}
$$

Assuming ideal gas in the hollow fibers the partial pressure in the closed end is given by

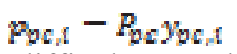

In hollow fibers, it is difficult to apply numerical modeling on the pressure drop. A methodology adopted by several researchers is to use Hagen-Poiseuille equation in a differential form to define the pressure change within the hollow fibers (Antonson, Gardner, King, \& Ko, 1977; Chern et al., 1985; C.Y. Pan \& Habgood, 1978).

$$
P \frac{d p_{p}}{d I}=\frac{128 \mu F_{s} T}{\pi N D_{1}^{d} T_{s}} n_{p}(l)
$$


The inner and outer diameter require in this numerical approach. For membrane modules working at highpressure differences, it looks acceptable to adopt that the pressure drop within the hollow fibers is insignificant equated to the driving forces. Therefore

$$
P_{\mathrm{sc}}=P_{\mathrm{b}}
$$

In a binary mixture, the resulting explicit equation on a differential area element for the permeate can be formulated (Giglia, Bikson, Perrin, \& Donatelli, 1991)

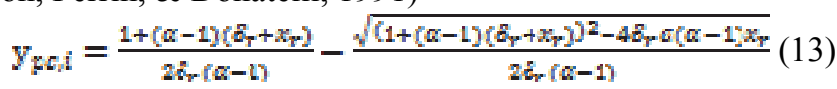

This expression is derived from the conditions of counter-current flow. At the close end of the hollow fiber, explicit expressions for the permeate purity have not been obtained for multicomponent mixtures. Established on the reasons published by Weller and Steiner (Weller \& Steiner, 1950), it can be presented that the resulting expression should exist between the components:

Equations (6), (9) and (12) jointly give

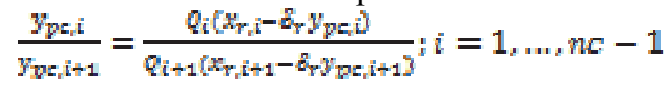

$$
n_{f} \theta y_{b i}=Q_{i} A \Delta P_{i=m} ; i=1, \ldots, n c
$$

Equation (7) defined $\Delta P_{i, l}$. By introducing the stage, cut changes the material balance and equation (8) becomes dimensionless.

$$
\begin{aligned}
& x_{f i i}=\theta y_{b, i}+(1-\theta) x_{r i} ; i=1, \ldots n c-1
\end{aligned}
$$

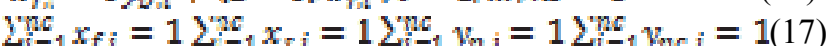

The equations (14) - (17) include a complete numerical model of a membrane, containing algebraic

Table 1. Operating parameters for numerical modeling

\begin{tabular}{lcccccc}
\hline Component & Helium & $\begin{array}{l}\text { Carbon } \\
\text { dioxide }\end{array}$ & Nitrogen & Methane & Ethane & Propane \\
\hline Symbol & $\mathrm{He}$ & $\mathrm{CO}_{2}$ & $\mathrm{~N}_{2}$ & $\mathrm{CH}_{4}$ & $\mathrm{C}_{2} \mathrm{H}_{6}$ & $\mathrm{C}_{3} \mathrm{H}_{8}$ \\
\hline $\begin{array}{l}\text { Mole } \\
\text { fraction (\%) }\end{array}$ & 0.82 & 1 & 15 & 71.1 & 6.4 & 5.68 \\
\hline $\begin{array}{l}\text { Permeability } \\
(\text { barrer) }\end{array}$ & 7.79 & 0.27 & 0.044 & 0.041 & 0.024 & 0.017 \\
\hline a 1 barrer $=1 \times 10^{-10} \mathrm{~cm}^{3}(\mathrm{STP}) \mathrm{cm} \mathrm{cm}^{-2} \mathrm{~s}^{-1} \mathrm{~cm} \mathrm{Hg}^{-1}$. & &
\end{tabular}

equations. This numerical model could not be applied on the shell side to guess the pressure drop of the membrane module. The pressure drop in shell side will usually be less than 1 bar in the case of commercial membrane modules, and in most cases, the default value of 0.3 bars may be applied. It is of immense significance for the numerical model to provide steady initial start approximations with robust and fast convergence. The above numerical model can be redeveloped by implementing the Paterson approximation to the logarithmic mean (Paterson, 1984). By using stage cut, retentate and permeate concentration can be obtained. The results obtained by this approach can be considered as the starting point

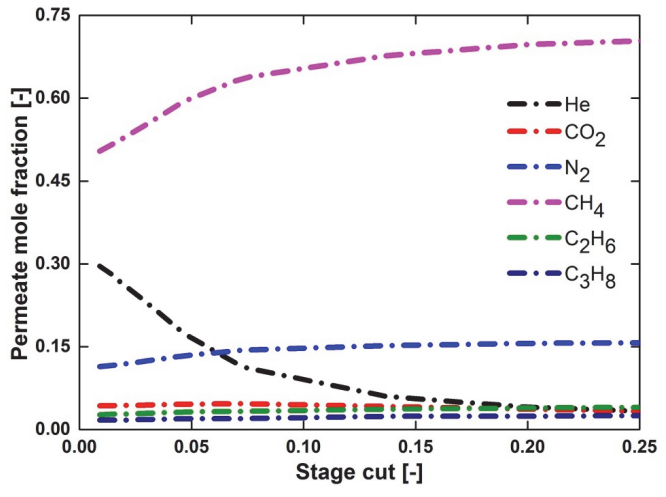

Figure 3. Permeate mole fraction as a function of stage cut for the solution or more optimization of the numerical technique for further conditions.

\section{RESULTS AND DISCUSSION}

The proposed numerical model has been applied to the data published in the literature. The operating parameters for cellulose acetate membrane hollow fiber with countercurrent flow configuration are summarized in Table 1 (Morita et al., 2007; Naghsh, Sadeghi, Moheb, Chenar, \& Mohagheghian, 2012; C. Y. Pan, 1983). Feed flow rate is changed to obtain different values of stage cut for the evaluation of membrane module. Figure 3 shows the concentration of helium and other components in permeate stream against stage cut, obtained by using above mentioned numerical methods. The ratio of total permeate flow to total feed 
flow is known as the stage cut. The helium losses in the retentate stream can be cut to a very low level by operating the membrane separation step at high stage-cut. Figure 4 shows two membrane separators in four different operating modes with different permeate pressure configurations. The permeate pressure in the permeator is assumed to be uniform, but different for each permeator (either $100 \mathrm{kPa}$ or $300 \mathrm{kPa}$ ). In all modes, the retentate stream for the first stage serves as a feed for the second stage.

In Mode $\mathrm{A}$, the feed gas with lower permeate pressure enters the unit, and the retentate is treated by the unit with a higher permeate pressure. This is the intermediate operating mode as shown by the permeate concentration and helium recovery shown in the figure. Reverse feed flow is applied to operate mode $\mathrm{B}$, this mode appears as most efficient one in terms of both helium permeation and recovery. In mode $\mathrm{C}$ permeator is operated with the same feed and retentate concentrations with different feed flow rates, has the less permeation value of helium as compared to mode B. Feed stream with high flow rate entered in low permeate pressure separator. Mode D operates high flow stream with high permeate pressure separator. Feed stream with high flow rate entered in high permeate pressure separator. This mode is operated like mode $\mathrm{C}$. The process of the membrane gas separation can reach significant decreases in helium loss with a single membrane separation stage. More stages or steps may optionally be used as preferred to increase the concentration of helium in permeate or otherwise adjust the composition of permeate or retentate streams. Selectivity and permeability of the membrane eventually play an important role in the permeation of the desired gas. Multistage membrane separators are used to achieve the certain level of helium in the permeate stream. This process is shown in Figure 5. This configuration is simulated by increasing stage cut at the same pressures in feed and permeates side for all three membrane separators. The higher product concentration and permeation rate are achieved at the low-pressure ratio. Same pressures on both sides of the membrane are maintained with the use of a compressor to achieve higher driving force. The permeate from the first stage serves as the feed to the second stage and similarly permeate of second stage acts as the feed to the third stage. Table 2 describes the values of streams shown in Figure 5. It is possible to achieve a permeate stream with $99.8 \%$ helium in three stages as shown in stream 7. The results presented in Table 2 shown that the enrichment of helium is possible with multi-stage separation.

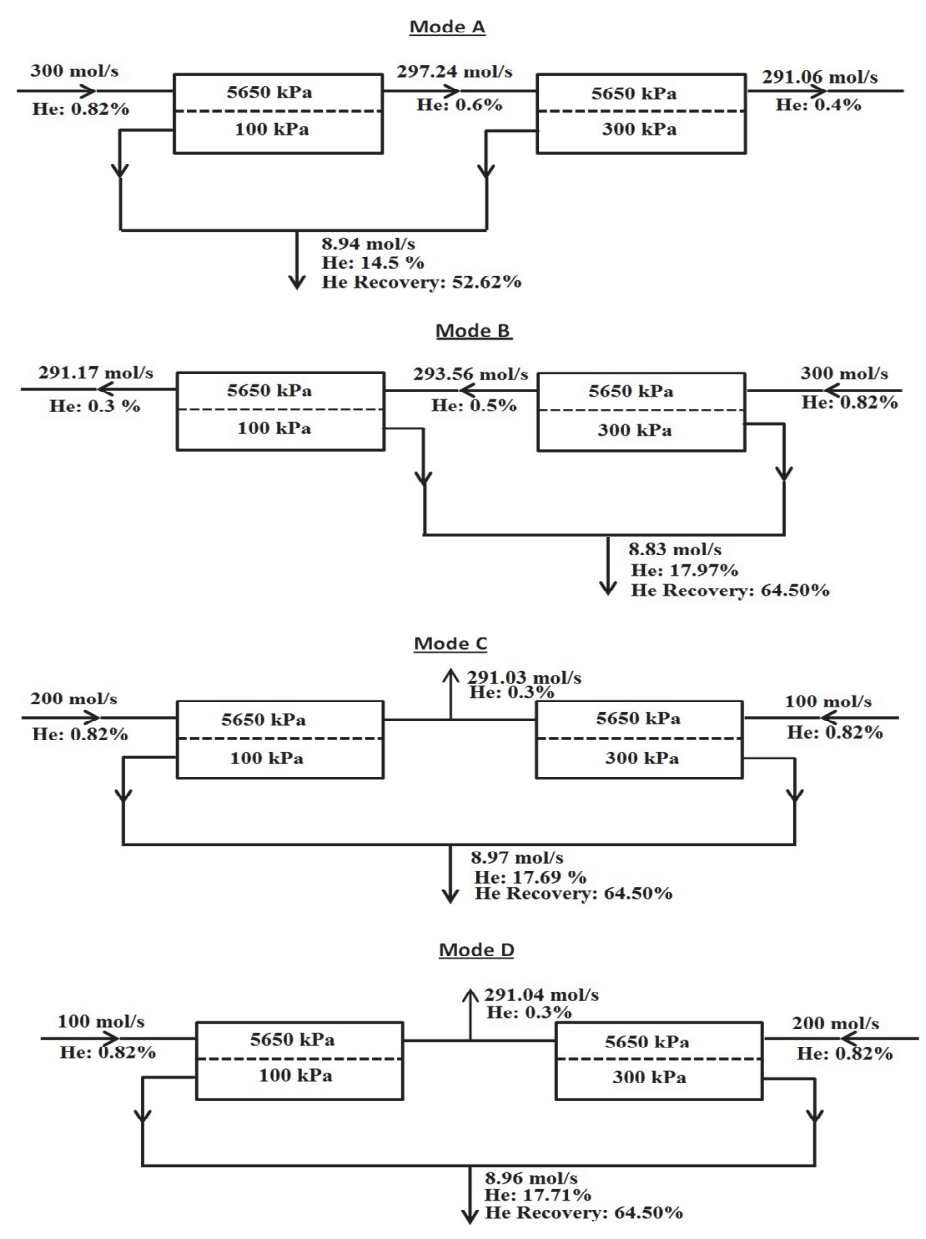

Figure 4. Effect of permeate pressure variation on permeation in multistage membrane separator

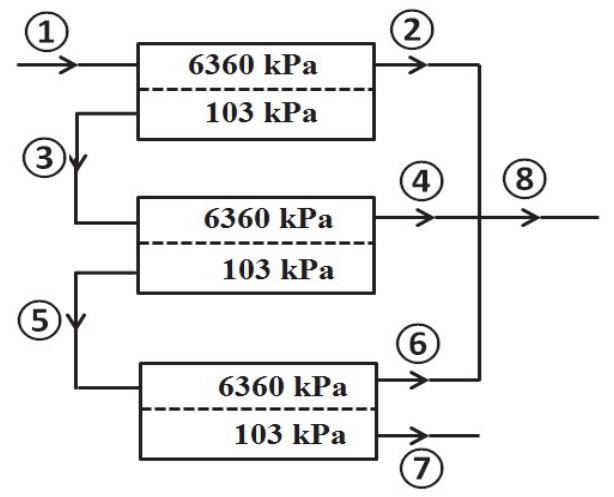

Figure 5. Multistage membrane permeation of helium from $0.82 \%$ feed

\section{CONCLUSIONS}


The numerical model proposed in this research work has advantages over other models commonly used. It requires less computational effort and time resulting in improved solution stability. Additionally, the computational complexity does not increase as the number of separation stages increases. The model predicted the helium separation and recovery from multiple components gas mixture with different flow rates quite adequately. By observing the results of the mathematical model, Table 2. Values of streams shown in Figure 5 it was found that the membrane can recover up to $99.9 \%$ helium in the permeate stream at a high stage cut. The countercurrent flow pattern predicted the high permeate mole fraction with minimum membrane area. This flow pattern is used to study the effect of different variables on separation of helium from the gas mixture. These variables included flow rate and composition of the feed, the pressure difference between the membrane and the total membrane area. The model is applicable to both single and multi-stage separation. Different modes are compared at different permeation pressure to predict a most efficient operating mode. The two or more separators at increasing pressure ratio and stage cut can be used to increase the overall performance of helium permeation, especially when a high concentration of the helium is needed.

\section{NOMENCLATURE}

A membrane area

Di inner diameter

Do outer diameter

d membrane thickness

$\mathrm{F}$ feed rate (local)

$\tilde{F}$ dimensionless feed rate $\tilde{F}=F / n \_f$

$\mathrm{N}$ number of fibers in a module, $\overline{\mathrm{N}}=\mathrm{A} /(\pi$ Do $\mathrm{L})$

$\mathrm{n}$ flow rate

nc number of components

$\mathrm{L}$ length of a fiber

1 coordinate of length

$\tilde{I}$ dimensionless coordinate of length, $\tilde{I}=1 / \mathrm{L}$

Pi permeability constant

$\mathrm{P}$ pressure

pi partial pressure

Qi overall permeability, $\mathrm{Qi}=\mathrm{Pi} / \mathrm{d}$

$\mathrm{R}$ permeation factor (Eq. 4)
$\mathrm{T}$ temperature

xi molar fraction (high pressure side)

yi molar fraction (low pressure side)

$\alpha \mathrm{i}$ selectivity of a membrane, $\alpha \mathrm{i}=\mathrm{Qi} / \mathrm{Qi}=\mathrm{nc}$

$\delta$ trans-membrane pressure ratio, $\delta=\mathrm{Pp} / \mathrm{Pf}$

$\theta$ stage cut (cut ratio), $\theta=\mathrm{np} / \mathrm{nf}$

$\mu$ viscosity of gas mixture

$\mathrm{g}$ trans-membrane pressure ratio, $\mathrm{g}=\mathrm{Pp} / \mathrm{Pf}$

$\Delta \mathrm{Plm}, \mathrm{i}$ logarithmic mean partial pressure difference, (Eq. 7)

\section{Subscripts}

f feed inlet (high pressure side)

$r$ retentate (residue) outlet (high pressure side)

$\mathrm{s}$ reference condition

p permeate outlet (low pressure side)

pc closed end of the hollow fiber

i component i, i $=1,2,3, \ldots$, nc.

\section{REFERENCES}

Ahmad, F., Lau, K., Lock, S., Rafiq, S., Khan, A. U., \& Lee, M. (2015). Hollow fiber membrane model for gas separation: Process simulation, experimental validation and module characteristics study. Journal of Industrial and Engineering Chemistry, 21, 1246-1257.

Ahsan, M., \& Hussain, A. (2014). Comparing numerical methods for multicomponent gas separation by single permeation unit. Chiang Mai Journal of Science, 41(1), 184-199.

Ahsan, M., \& Hussain, A. (2015). Mathematical modeling of helium recovery from a multicomponent fuel gas with polymeric membrane. International Journal of Chemical Engineering and Applications, 6(3), 173178.

Antonson, C. R., Gardner, R. J., King, C. F., \& Ko, D. Y. (1977). Analysis of gas separation by permeation in hollow fibers. Industrial \& Engineering Chemistry Process Design and Development, 16(4), 463-469. doi: 10.1021/i260064a005

Barnicki, S. D., \& Fair, J. R. (1992). Separation system synthesis: a knowledge-based approach. 2. Gas/vapor mixtures. Industrial \& Engineering Chemistry Research, 31(7), 1679-1694. 
Basaran, O. A., \& Auvil, S. R. (1988). Asymptotic analysis of gas separation by a membrane module. AIChE Journal, 34(10), 1726-1731. doi: 10.1002/aic.690341019

Boucif, N., Majumdar, S., \& Sirkar, K. K. (1984). Series solutions for a gas permeator with countercurrent and cocurrent flow. Industrial \& Engineering Chemistry Fundamentals, 23(4), 470-480. doi: 10.1021/i100016a016

Chern, R. T., Koros, W. J., \& Fedkiw, P. S. (1985). Simulation of a hollow-fiber gas separator - the effects of process and design variables. Industrial \& Engineering Chemistry Process Design and Development, 24(4), 1015-1022. doi: 10.1021/i200031a020

Chowdhury, M. H. M., Feng, X. S., Douglas, P., \& Croiset, E. (2005). A new numerical approach for a detailed multicomponent gas separation membrane model and AspenPlus simulation. Chemical Engineering \& Technology, 28(7), 773-782. doi: 10.1002/ceat.200500077

Coker, D. T., Freeman, B. D., \& Fleming, G. K. (1998). Modeling multicomponent gas separation using hollow-fiber membrane contactors. AIChE Journal, 44(6), 1289-1302. doi: 10.1002/aic.690440607

Fattah, K. A., Hamam, S. M., Alenezi, G., Ettoueny, H. M., \& Hughes, R. (1992). A nonideal model for analysis of gas separation permeators. Journal of Membrane Science, 65(3), 247-257. doi: 10.1016/03767388(92)87026-t

Fritzsche, A., \& Narayan, R. (1987). Gas separation by membrane systems. Chem. Economy and Eng. Rev, 19(205), 19-31.

Giglia, S., Bikson, B., Perrin, J. E., \& Donatelli, A. A. (1991). Mathematical and experimental-analysis of gas separation by hollow fiber membranes. Industrial \& Engineering Chemistry Research, 30(6), 12391248. doi: 10.1021/ie00054a026

Hogsett, J., \& Mazur, W. (1983). Estimate membrane system area. Hydrocarbon processing, 62(8), 52-54.

Kaldis, S. P., Kapantaidakis, G. C., \& Sakellaropoulos, G. P. (2000). Simulation of multicomponent gas separation in a hollow fiber membrane by orthogonal collocation - hydrogen recovery from refinery gases. Journal of Membrane Science, 173(1), 61-71. doi: 10.1016/s0376-7388(00)00353-7

Krovvidi, K. R., Kovvali, A. S., Vemury, S., \& Khan, A. A. (1992). Approximate solutions for gas permeators separating binary-mixtures. Journal of Membrane Science, 66(2-3), 103-118. doi: 10.1016/0376-7388(92)87001-e

Kvaalen, E. (1991). A faster Broyden method. BIT Numerical Mathematics, 31(2), 369-372.

Lock, S., Lau, K., \& Shariff, A. (2015). Effect of recycle ratio on the cost of natural gas processing in countercurrent hollow fiber membrane system. Journal of Industrial and Engineering Chemistry, 21, 542551 .

Meindersma, G. (1990). Comparison of several hydrogen separation processes. Paper presented at the Process Technology Proceedings.

Morita, R., Khan, F. Z., Sakaguchi, T., Shiotsuki, M., Nishio, Y., \& Masuda, T. (2007). Synthesis, characterization, and gas permeation properties of the silyl derivatives of cellulose acetate. Journal of Membrane Science, 305(1), 136-145.

Naghsh, M., Sadeghi, M., Moheb, A., Chenar, M. P., \& Mohagheghian, M. (2012). Separation of ethylene/ethane and propylene/propane by cellulose acetate-silica nanocomposite membranes. Journal of Membrane Science, 423, 97-106.

Pan, C. Y. (1983). Gas separation by permeators with high-flux asymmetric membranes. AIChE Journal, 29(4), 545-555. doi: 10.1002/aic.690290405

Pan, C. Y., \& Habgood, H. (1978). Gas separation by permeation Part II: Effect of permeate pressure drop and choice of permeate pressure. The Canadian Journal of Chemical Engineering, 56(2), 210-217.

Paterson, W. R. (1984). A replacement for the logarithmic mean. Chemical Engineering Science, 39(11), 1635-1636. doi: 10.1016/0009-2509(84)80090-1

Pettersen, T., \& Lien, K. M. (1994). A new robust design model for gas separating membrane modules, based on analogy with counter-current heat exchangers. Computers and Chemical Engineering, 18(5), 427-439.

Rautenbach, R., \& Dahm, W. (1986). Simplified calculation of gas-permeation hollow-fiber modules for the separation of binary-mixtures. Journal of Membrane Science, 28(3), 319-327. doi: 10.1016/s03767388(00)82042-6

Scholz, M., Alders, M., Lohaus, T., \& Wessling, M. (2015). Structural optimization of membrane-based biogas upgrading processes. Journal of Membrane Science, 474, 1-10.

Spillman, R. W. (1989). Economics of gas separation membranes. Chemical Engineering Progress, 85(1), 4162.

Stookey, D., Patton, C., \& Malcolm, G. (1986). Membranes separate gases selectively. Chemical Engineering Progress, 82(11), 36-40.

Weller, S., \& Steiner, W. A. (1950). Separation of gases by fractional permeation through membranes. Journal of Applied Physics, 21(4), 279-283. doi: 10.1063/1.1699653 